\title{
Constraints on the radiation temperature before inflation
}

\author{
Ramón Herrera a,, Diego Pavónb,2 and Joel Saavedrac3 \\ ${ }^{1}$ Instituto de Física. Pontificia Universidad Católica \\ de Valparíso, casilla 4056, Valparaíso, Chile \\ ${ }^{2}$ Departmento de Física, Universidad Autónoma de Barcelona, \\ 08193 Bellaterra (Barcelona), Spain \\ ${ }^{3}$ Pontificia Universidad Católica de Valparíso, Chile
}

\begin{abstract}
We consider the short period of cosmic expansion ranging from the end of the Planck era to the beginning of inflation and set upper and lower limits on the temperature of the radiation at the commencement of the inflationary phase.
\end{abstract}

\footnotetext{
${ }^{a}$ E-mail: ramon.herrera@pucv.cl

b E-mail: diego.pavon@uab.es

${ }^{\text {c }}$ E-mail: joel.saavedra@pucv.cl
} 


\section{INTRODUCTION}

Nowadays it is widely accepted that the homogeneity and isotropy we observe today at cosmic scales, as well as the seeds of the present matter structure, can be explained by a short period of very rapid expansion experienced by the primeval universe. According to the common lore this expansion was driven by a scalar field (not yet identified), whose nearly constant potential dominated every other form of energy during the aforesaid expansion (see [1] for a comprehensive review), though other mechanisms responsible for inflation are conceivable [2, 3]. Either way, this expansion erased, or nearly erased, any information we could otherwise acquire about the state of the universe before inflation set in. Nevertheless it is obvious that the main energy component in the interval between the end of the Planck era and the commencement of the inflationary period fulfilled all the energy conditions and it seems natural to identify it as radiation (photons and neutrinos) plus ultrarelativistic matter, all of them at thermal equilibrium with each other at temperature $T_{\gamma}$. The latter goes down with expansion as the inverse of the scale factor of the Friedmann-RobertsonWalker (FRW) metric. Ihis paper aims to set limits (lower and upper bounds) on that temperature at the onset of inflation.

To do this we shall assume that: (i) The inflationary expansion began below the Planck energy scale. (ii) Inflation was driven by some scalar field, $\phi$, that violated the energy conditions, had vanishing entropy as it was in a pure quantum state. (iii) During that short inflationary period the scalar potential, $V(\phi)$, was the dominant energy component. (iV) Between the Planck regime and the beginning of inflation, radiation plus ultrarelativistic particles dominated the expansion.

Clearly, the said temperature limits are to depend on the inflaton field. We shall consider two well motivated cold inflationary scenarios consistent with the 2015 Planck's data [4].

First the chaotic model [5]; secondly, Chiba's model [6]. The latter covers several interesting models as limiting cases and it is rater general.

In both models we shall resort to the generalized second law of thermodynamics. This law was introduced in connection with the thermodynamics of black holes [7] and it is a 
straightforward extension to gravitational physics of the ordinary second law when the black hole entropy is considered. It lays that the entropy of a black hole (one quart of the area of its event horizon) plus the entropy of its surroundings cannot diminish. In cosmic settings, and in the absence of black holes, it has been formulated by saying that the entropy of the cosmic horizon (either the future event horizon or the apparent horizon) plus the entropy of matter and fields inside the horizon cannot decrease [8]. Recently, it has been applied in the study of the late evolution of some FRW models [9], and in the analysis of the viability of nonsingular bouncing universe models [10].

Here we shall consider the apparent horizon, defined as the boundary hypersurface of the antitrapped spacetime region, since it always exists both for expanding and contracting universes. By contrast, the future event horizon exists only if the universe accelerates for $t \rightarrow \infty$. A spacetime region is called antitrapped if the ingoing and outgoing radial null geodesics have positive expansion (see [11] and [12] for details). As a consequence the radius of the apparent horizon (centered at the comoving observer position) reads $r_{H} \equiv$ $\left(H^{2}+k a^{-2}\right)^{-1 / 2}$, where $k$ denotes the scalar curvature index of the FRW metric and, as usual, $H=\dot{a} / a$, the Hubble factor. The entropy of the horizon, proportional one quarter of the area of the horizon, is simply $S_{H}=\pi r_{H}^{2} / l_{\mathrm{p}}^{2}$ in units of the Boltzmann constant, with $l_{\mathrm{p}}$ the Planck length. At the primeval epochs we are interested in (before inflation and just after it) $a \ll 1$ while $H$ is large, thereby the horizon entropy is excellently approximated by $S_{H}=\pi l_{\mathrm{p}}^{-2} H^{-2}$. In this work we shall use units such that $k_{B}=c=\hbar=1$.

\section{UPPER AND LOWER BOUNDS ON THE TEMPERATURE AT THE BEGIN- NING OF COLD INFLATION}

Let us consider that inflation started somewhat below the Planck energy scale. Then, it is reasonable to assume that before the inflaton field dominated the expansion this was driven by some other energy fields that comply with the null, dominant and strong energy conditions. Let us model these fields as thermal radiation characterized by some common temperature $T_{\gamma} \propto a^{-1}$.

Let the de Sitter inflation $\left(H=\right.$ constant) begin at $t=t_{1}$ and end at $t=t_{\text {end }}$, - see Fig. 11, 
The radiation entropy inside the apparent horizon at $t_{1}$ will be $S_{\gamma}\left(t_{1}\right)=K_{2} T_{\gamma 1}^{3} / H_{1}^{3}$, where $K_{2}=(4 \pi / 3)\left(2 \pi^{2} g_{*} / 45\right)$ with $g_{*}$ the number of relativistic degrees of freedom, approximately 100 in our case. Because of the temperature redshift, $T_{\gamma \text { end }}=T_{\gamma 1} / \exp \left(H\left(t_{\text {end }}-t_{1}\right)\right) \ll T_{\gamma 1}$, the corresponding entropy at $t_{\text {end }}$ will be negligible as compared to $S_{\gamma}\left(t_{1}\right)$. On the other hand, since $H_{1}=H_{\text {end }}$, in this inflationary scenario the total entropy (i.e., the entropy of the apparent horizon, $\propto H^{-2}$, plus the entropy of the radiation inside the horizon) will have diminished at $t_{\text {end }}$ by about $S_{\gamma}\left(t_{1}\right)$. Bearing in mind the generalized second law, this means that the expansion rate, $H$, cannot be exactly constant during inflation. It must experience a slight decrease so that $H\left(t_{\text {end }}\right)$ should be in reality $H_{\text {end }^{\prime}}=H_{1}(1-x)$ with $0<x \ll 1$, a small number fixed by the values of $H_{1}$ and $H_{\text {end' }}$.

From $\Delta S_{H} \geq \Delta S_{\gamma}$ it follows that

$$
\frac{\pi}{l_{\mathrm{p}}^{2}}\left(\frac{1}{H_{\mathrm{end}^{\prime}}^{2}}-\frac{1}{H_{1}^{2}}\right) \geq K_{2} \frac{T_{\gamma 1}^{3}}{H_{1}^{3}} .
$$

If the inflationary expansion is driven by a scalar field, $\phi$, that slowly rolls-down its potential $V(\phi)$, with the help of Friedmann's equation $H^{2}=\kappa V(\phi)$, Eq. (11) can be recast as

$$
T_{\gamma 1} \leq\left\{2 \frac{K_{1}}{K_{2}} \sqrt{\kappa}\left[V^{1 / 2}\left(\phi_{i}\right)-V^{1 / 2}\left(\phi_{\text {end }}\right)\right] m_{p}^{2}\right\}^{1 / 3},
$$

where $\kappa=8 \pi /\left(3 m_{p}^{2}\right)$ (recall that in our units $\left.G=l_{p}^{2}=1 / m_{p}^{2}\right), K_{1}=2 \pi$, and $K_{2}=183.46$ for $g_{*}=100$. We have substituted $\phi\left(t_{i}\right)$ by $\phi\left(t_{1}\right)$ where $t_{i} \gtrsim t_{1}$ is the time at which the radiation has redshifted by a factor of 100 , which corresponds to approximately 5 e-folds. At that time the energy density is already overwhelmingly dominated by the scalar field. Not so at $t_{1}$ when inflation began, because at that time the energy density of the radiation - though lower than $V\left(\phi_{1}\right)$ —was, however, of the same order.

To go from (11) to (2) we used $H_{1} x=H_{1}-H_{\mathrm{end}^{\prime}}$ as well as

$$
H_{1}^{3}\left(\frac{1}{H_{\mathrm{end}}^{2}}-\frac{1}{H_{1}^{2}}\right) \simeq 2 H_{1} x \quad(0<x \ll 1) .
$$

Note that $x=1-\sqrt{V\left(\phi_{\text {end }}\right) / V\left(\phi_{i}\right)}$. Also, $H_{1} \gtrsim H_{i}=\sqrt{\kappa V_{i}(\phi)}$, and $H_{\text {end }^{\prime}}=\sqrt{\kappa V_{\text {end }}(\phi)}$. Next we apply Eq. (2) first to the chaotic model of inflation and then to Chiba's model. 


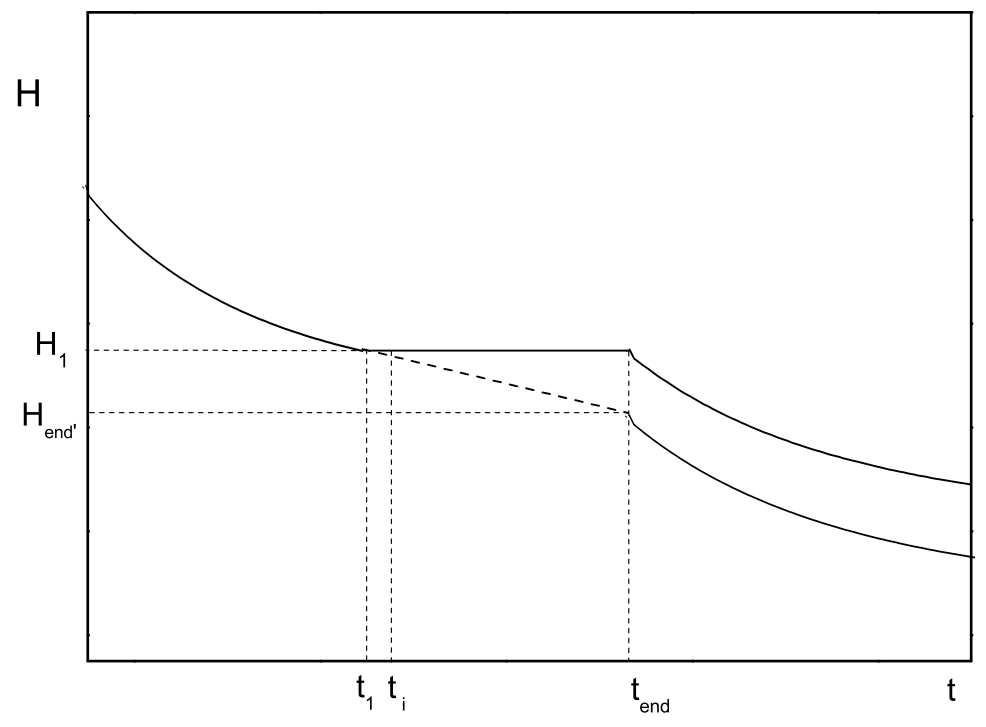

Figure 1. Schematic evolution of the Hubble factor with time. Inflation starts at $t_{1}$ and ends at $t_{\text {end }}$. Before $t_{1}$ the universe was dominated by fields that fulfill the energy conditions whence $\dot{H}<0$. If the inflationary expansion were exactly de Sitter (solid horizontal line), then $H_{1}=H_{\text {end }}$. The long-dashed line (connecting $\left(t_{1}, H_{1}\right)$ and $\left.\left(t_{\text {end }}, H_{\text {end }^{\prime}}\right)\right)$ describes more realistically the inflationary stage rendering it now compatible with the second law (see the main text). In this scenario, the entropy of the apparent horizon augments during this epoch. The instant $t_{i}$ corresponds to the time when the energy density of radiation has declined due to the inflationary expansion by a factor of, say, 100 -about 5 e-folds. From $t_{\text {end }}$ onwards the universe gets dominated by the matter and radiation generated nearly instantaneously in the reheating, again $H \propto t^{-1}$.

\section{A. Chaotic inflation}

The simplest chaotic inflation model [5] is characterized by the potential

$$
V(\phi)=\frac{1}{2} m^{2} \phi^{2}
$$

where $m$ stands for the inflaton mass. To set an upper bound on $T_{\gamma}$ at the beginning of inflation it is expedient to express this potential in terms of the number of e-folds. The latter is given by

$$
N=\int_{t_{1}}^{t_{\mathrm{end}}} H d t=3 \kappa \int_{\phi_{\mathrm{end}}}^{\phi_{i}} \frac{V}{V_{, \phi}} d \phi .
$$


Therefore,

$$
\frac{m^{2}}{2} N=\frac{3}{4}\left[H_{i}^{2}-H_{\mathrm{end}^{\prime}}^{2}\right], \quad \phi_{\mathrm{end}}=\frac{2}{\sqrt{6 \kappa}},
$$

and

$$
H_{i}=\sqrt{\frac{2}{3} m^{2}\left(N+\frac{1}{2}\right)} \lesssim H_{1},
$$

Consequently, assuming $N=60$ we have from Eq. (2) with $K_{1} / K_{2} \simeq 0.0343$

$$
T_{\gamma 1} \leq\left\{2 \times 0.034\left[\sqrt{\frac{2}{3}\left(60+\frac{1}{2}\right)}-\frac{1}{\sqrt{3}}\right] m m_{\mathrm{p}}^{2}\right\}^{1 / 3} .
$$

On the other hand, Planck's experiment yields $P_{s} \sim 10^{-9}$ for the power of scalar modes at the end of inflation. This for the present model implies $m \simeq 10^{-6} m_{\mathrm{p}}[1]$. Plugging this into (6) leads to an upper bound on the radiation temperature at the start of inflation, namely, $T_{\gamma 1} \leq 7.3 \times 10^{-3} m_{\mathrm{p}}$

A lower bound on $T_{\gamma 1}$ can be obtained as follows. Inflation began when $\ddot{a}$ became positive, i.e., when $\rho_{\gamma}+\rho_{\phi}+3\left(p_{\gamma}+p_{\phi}\right)$ vanished. Recalling that at $t=t_{1}$ one had that $(\dot{\phi})^{2} / 2$ was negligible against $V(\phi)$ and $\rho_{\phi}$, because of the slow roll, and that $p_{\gamma}=\rho_{\gamma} / 3, \rho_{\phi} \simeq V(\phi)$, $p_{\phi} \simeq-V(\phi)$, this occurred when $\rho_{\gamma}=V(\phi)$, i.e., $\rho_{\gamma 1}=V\left(\phi_{1}\right)$. On the other hand, $V\left(\phi_{1}\right)>$ $V\left(\phi_{\text {end }}\right)$. Therefore $\rho_{\gamma 1}>V\left(\phi_{\text {end }}\right)$; that is to say,

$$
\frac{\pi^{2}}{30} g_{*} T_{\gamma 1}^{4}>\frac{1}{2} m^{2} \phi_{\text {end }}^{2}=\frac{1}{3} \frac{m^{2}}{\kappa} .
$$

Keeping in mind that $m \simeq 10^{-6} m_{\mathrm{p}}$ and that $g_{*}=100$, we get the lower bound $T_{\gamma 1}>1.8 \times 10^{-4} m_{\mathrm{p}}$.

Combining this with the upper bound derived above, we can write $1.8 \times 10^{-4} m_{\mathrm{p}}<T_{\gamma 1}<7.3 \times 10^{-3} m_{\mathrm{p}}$.

\section{B. Chiba's model}

In this model the potential expressed in terms of the number of e-folds reads [6]

$$
V(N)=\frac{N}{\alpha+\beta N},
$$


where $\alpha$ and $\beta$ are integration constants with $\alpha>0$ and $\beta<\alpha$ except that $\beta \neq 0$. Both constants share units of $m_{\mathrm{p}}^{-4}$. The meaning of these becomes clear when one realizes that $\alpha=-N^{2} d\left(V^{-1}\right) / d N$ and $\beta=\lim _{N \rightarrow \infty} V^{-1}$. Note that, at variance with most models of slow-roll, $V$ increases with $N$ (i.e., $\left.d V(N) / d N=\alpha /(\alpha+\beta N)^{2}>0\right)$. Next we briefly consider the cases $\beta>0$ and $\beta<0$. The case $\beta=0$ reduces to the chaotic model above considered - see [6] for details.

For $\beta>0$ the number of e-folds can be written in terms of the inflaton field as

$$
N(\phi)=\frac{1}{\gamma^{2}} \sinh ^{2}\left[\frac{\sqrt{3 \kappa} \gamma}{2}(\phi-C)\right]
$$

where the parameter $\gamma$ is defined by $\gamma \equiv \sqrt{|\beta| / \alpha}$ and $C$ is an integration constant. With the help of (8) last equation produces

$$
V(\phi)=\frac{1}{\beta} \tanh ^{2}[\sqrt{3 \kappa} \gamma(\phi-C) / 2]
$$

This is known as the "T-model" potential of Kallosh and Linde [13]. Likewise, when $\gamma=\sqrt{2 / 3}$ it reduces to Starobinsky's model [2], and to the $\alpha$-attractor model [13] for $\alpha=2 /\left(3 \gamma^{2}\right)$.

For $\beta<0$ one has $N=\gamma^{-2} \sin ^{2}[(\sqrt{3 \kappa} / 2)(\phi-C)]$, hence the condition $\gamma^{2} \ll 1$ must be fulfilled for an efficient inflation. This combined with Eq. (8) yields $V(\phi) \simeq(4 \alpha)^{-1} 3 \kappa(\phi-C)^{2}$, i.e., the quadratic potential.

Since, contrary to the chaotic-inflation case, $d N / d|\phi|>0$, when using Eq. (2) the order of the terms within the square parenthesis must be reversed

$$
T_{\gamma 1} \leq\left\{2 \frac{K_{1}}{K_{2}} \sqrt{\kappa}\left[V^{1 / 2}\left(N_{\text {end }}\right)-V^{1 / 2}\left(N_{i}\right)\right] m_{\mathrm{p}}^{2}\right\}^{1 / 3} .
$$

Using last expression alongside Eq. (8) and choosing $N_{i}=5$ and $N_{\text {end }}=60$ we can write

$$
\alpha^{1 / 6} T_{\gamma 1} \leq\left\{0.0343 \sqrt{\frac{8 \pi}{3}}\left[\sqrt{\frac{60}{1+\gamma^{2} 60}}-\sqrt{\frac{5}{1+\gamma^{2} 5}}\right]\right\}^{1 / 3} m_{p}^{1 / 3} .
$$


To estimate $\gamma^{2}$ we exploit the fact that in the T-model the tensor to scalar ratio, $r \equiv P_{T} / P_{S}$, between the power of tensorial and scalar modes generated at the end of inflation is given by [6]

$$
r= \begin{cases}\frac{8}{N_{\text {end }}+\gamma^{2} N_{\text {end }}^{2}} & (\beta>0) \\ \frac{8}{N_{\text {end }}} & (\beta \leq 0) .\end{cases}
$$

This combined with the result from the Keck Array and BICEP collaborations [14] about the said ratio, namely $r_{0.05}<0.07$ at $95 \%$ confidence level, yields $\gamma^{2}<1.50793 \times 10^{-2}$ for $N_{\text {end }}=60$.

Now, taking into account that $V\left(\phi_{\text {end }}\right) \simeq 10^{-12} m_{\mathrm{p}}^{4}[4]$ and that $V(N=60) \simeq 10^{2} / \alpha$ it follows that $\alpha \sim 10^{14} m_{\mathrm{p}}^{-4}$.

Inserting the above estimation of $\alpha$ and the upper bound on $\gamma^{2}$ in Eq. (12) we obtain $T_{\gamma 1} \leq 3 \times 10^{-3} m_{\mathrm{p}}$. As it can be checked, this order of magnitude of the bound is rather insensible to the initial number of e-folds, $N_{i}$, provided it remains low (i.e., no larger than 10). Likewise as it can be verified, for the specific case of $\beta=0$ this upper bound remains practically unaltered.

To determine the lower bound on $T_{\gamma 1}$ we proceed as in the case of chaotic inflation and use the experimentally found value $V\left(\phi_{\text {end }}\right) \simeq 10^{-12} m_{\mathrm{p}}^{4}$; i.e.,

$$
\frac{\pi^{2}}{30} g_{*} T_{\gamma 1}^{4}>10^{-12} m_{\mathrm{p}}^{4} .
$$

This gives $T_{\gamma 1}>2.3 \times 10^{-4} m_{\mathrm{p}}$. In summary, the T-model implies $2.3 \times 10^{-4} m_{p}<T_{\gamma 1}<3.05 \times 10^{-3} m_{\mathrm{p}}$. This interval is similar to the one obtained in the case of the chaotic model.

Equations (8) and (13,a) combined with (11) lead to

$$
r \geq \frac{3 K_{2}^{2}}{32 \pi K_{1}^{2}} \frac{8 \alpha}{N_{\mathrm{end}}^{2}} T_{\gamma 1}^{6} .
$$

This expression provides us with an upper limit on the ratio $P_{T} / P_{S}$. In arriving to it we have neglected the term $V^{1 / 2}\left(N_{i}\right)$. The latter can be estimated as three times less than $V^{1 / 2}\left(N_{\text {end }}\right)$; obviously he approximation is not very good but we are just seeking a rough estimate for an upper bound on the said ratio. Inserting for $T_{\gamma 1}$ the upper bound found above and $N_{\text {end }}=60$ in (15) we find $r \geq 10^{-8}$. 


\section{DISCUSSION}

At the moment no empirical information is available about the short expansion phase between the end of the Planck era and the beginning of inflation. Maybe some will be gathered in the future once the the gravitational waves supposedly produced in that period are detected and successfully analyzed. Hence our current knowledge of that fleeting but crucial early epoch of the universe history is necessarily slim. This lends much interest to its study provided it is based on sound physics rather than just speculation.

In this paper, we resorted to the generalized second law of thermodynamics to set an upper bound on the temperature of the radiation at the beginning of the inflationary expansion. A lower bound was found by imposing that the energy density of the radiation at that instant, $t_{1}$, should be greater than that of the inflaton at $t_{\text {end }}$, see Fig. 1. We did this for the simplest chaotic model and Chiba's model which show compatibility with the constraints derived from the Planck's mission [4]. In both instances we got similar bounds on $T_{\gamma 1}$ (roughly in the range $\left(10^{-4}, 10^{-3} m_{\mathrm{p}}\right)$, consistent with the very reasonable assumption of being no lower than the reheating temperature. According to Chiba the latter lies in the interval $\left[10^{-8}, 10^{-4} m_{p}\right][6]$.

Likewise, we obtained an upper limit of the tensor to scalar ratio, Eq. (15). Though the bound is rather loose it, nonetheless, ensures that gravitational waves are indeed produced during inflation.

\section{ACKNOWLEDGMENTS}

One of us, DP, is indebted to the "Instituto de Física de la Pontificia Universidad Católica de Chile" for warm hospitality. This work was supported by the Agency CONICYT of the Chilean Government under contract MEC 80160077.

[1] B. Basset, S. Tsujikawa, and D. Wands, Rev. Mod. Phys. $\underline{78}, 537$ (2006).

[2] A.A. Starobinsky, Phys. Lett. B 1ㅜ, 99 (1980). 
[3] S. Capozziello, V. F. Cardone, H. Farajollahi and A. Ravanpak, Phys. Rev. D $\underline{84}, 043527$ (2011).

[4] Planck Collaboration, P.A.R. Ade et al., A \& A $\underline{594}$, A20 (2016).

[5] A.D. Linde, Phys. Lett. B $\underline{129}, 177$ (1983).

[6] T. Chiba, Prog. Theor. Exp. Phys. 2015, 073 E02.

[7] J.D. Bekenstein , Phys. Rev. D , 2333 (1973); $\underline{9}, 3292$ (1974).

[8] G. Gibbons and S.W. Hawking, Phys. Rev. D $\underline{15}, 2738$ (1977); P.C.W. Davies, Clasical Quantum Gravity $\underline{4}, \mathrm{~L} 225$ (1987); 모 1349 (1988); D. Pavón, Clasical Quantum Gravity $\underline{7}, 487$ (1990).

[9] J.P. Mimoso and D. Pavón, Phys. Rev. D $\underline{87}, 0473902$ (2013).

[10] P.C. Ferreira and D. Pavón, Eur. Phys. J. C $\underline{76}, 37$ (2016).

[11] D. Bak and S.-J. Rey, Class. Quantum Grav. 17, L83 (2000).

[12] R.G. Cai and L.M. Cao, Phys. Rev. D 포, 064008 (2007).

[13] R. Kallosh and A. Linde, J. Cosmology Particle Phys. 1307, 002 (2013).

[14] P.A.R. Ade et al. (Keck Array and BICEP Collaborations), Phys. Rev. Lett. 116, 031302 (2016). 